\title{
ANÁLISIS ENERGÉTICO DEL SISTEMA EXPERIMENTAL TQ PLINT TE94 QUE FUNCIONA COMO ENFRIADOR DE AIRE Y COMO CALEFACTOR
}

\section{EXPERIMENTAL SYSTEM TQ PLINT TE94 ENERGY ANALYSIS THAT WORKS AS AN AIR COOLER AND HEATER}

\author{
Eng. Emanuel López Alba*, PhD. Helen D. Lugo Méndez**, \\ MSc. Sergio Castro Hernández*, MSc. Edgar Vicente Torres González*, \\ MSc. Israel Eliú Hernández Mora*, MSc. Alejandro Torres Aldaco*, PhD. Raúl \\ Lugo Leyte*.
}

"Universidad Autónoma Metropolitana-Iztapalapa, Departamento de Ingeniería de Procesos e Hidráulica, Av. San Rafael Atlixco No. 186, Colonia Vicentina, 09340, Iztapalapa, CDMX, México. 00-52-55-58044600-Ext:2853. ela@xanum.uam.mx,sch@xanum.uam.mx, etorres@xanum.uam.mx, iehm@xanum.uam.mx, ata@xanum.uam.mx, lulr@xanum.uam.mx.

**Universidad Autónoma Metropolitana-Cuajimalpa, Departamento de Procesos y Tecnología, Av. Vasco de Quiroga No. 4871, Colonia Santa Fé, 05348, Cuajimalpa, CDMX, México. 00-52-55-58146500-Ext:3867. hlugo@correo.cua.uam.mx.

Resumen: El desempeño de las bombas de calor y de los enfriadores de aire está relacionado con la eficiencia y el consumo de energía. Es importante estudiarlos con el fin de determinar su mayor coeficiente de operación (COP). En este trabajo se realiza un análisis energético al enfriador de aire/bomba de calor experimental TQ PLINT TE94 con R22, en el cual, se varió el flujo de agua en 2, 4, 6 y $8 \mathrm{~L} / \mathrm{min}$. Para la bomba de calor, el COP bajo se debe al aumento de la diferencia de temperaturas entre el refrigerante y los fluidos externos (agua y aire); sin embargo, estas pérdidas son inevitables, además, los mayores COPs se obtuvieron para el flujo de agua de $8 \mathrm{~L} / \mathrm{min}$ con una potencia del compresor de $917 \mathrm{~W}$. Para un flujo de agua de $4 \mathrm{~L} / \mathrm{min}$ en el enfriador, se suministra al compresor la menor potencia y se obtuvieron los mayores COPs reales.

Palabras clave: Bomba de calor, Coeficiente de Operación, Enfriador de Aire, Refrigerante.

\begin{abstract}
The performance of heat pumps and air coolers is related to efficiency and energy consumption. Their study results relevant, in order to determine their best coefficient of performance. In this paper, an energy analysis of the experimental air cooler/heat pump TQ PLINT TE94 using R22 as the refrigerant is performed, in which the water flow is taken to be 2, 4, 6 and $8 \mathrm{~L} / \mathrm{min}$. For the heat pump, the low COP is due to the increase in the temperature difference between the refrigerant and the external fluids (water and air); however, these losses are unavoidable, in addition, the highest COP's are obtained for the water flow of $8 \mathrm{~L} / \mathrm{min}$ with a compressor power of $917 \mathrm{~W}$. For a water flow of $4 \mathrm{~L} / \mathrm{min}$ in the cooler, the lowest power is supplied to the compressor and the highest real COP's is obtained.
\end{abstract}

Keywords: Heat pump, Coefficient of Performance, Air Cooler, Refrigerant. 


\section{INTRODUCCIÓN}

Una de las principales aplicaciones de las bombas de calor por compresión de vapor es proporcionar calefacción y enfriamiento a un determinado espacio. El espacio puede ser pequeño, como el interior de un vehículo, hasta uno de mayor tamaño en donde el calor se transfiere de un cuarto a otro.

La tecnología de las bombas de calor para calefacción/enfriamiento no es nueva; sin embargo, todavía se desarrollan nuevas aplicaciones; por ejemplo, la calefacción y el enfriamiento, así como la provisión de agua caliente a una casa se pueden llevar a cabo simultáneamente (Chua et al., 2010). Con relación a su función y necesidades operativas, las bombas de calor pueden calentar y enfriar casas o edificios y se pueden dividir en cuatro categorías: (1) bombas de calor sólo para calefacción, que proveen calefacción y/o calentamiento de agua; (2) bombas de calor para calefacción y enfriamiento, que dan tanto calefacción como enfriamiento; (3) sistemas integrados de bomba de calor, que entregan calefacción, enfriamiento, calentamiento de agua y, a veces, recuperación de calor del aire de escape; y (4) calentadores de agua con bomba de calor, totalmente dedicados al calentamiento de agua (Saloux et al., 2018). La energía es un recurso limitado cuyo uso se debe hacer con alta eficiencia, bajo impacto medioambiental y al menor costo donde el consumo de energía eléctrica registrado dependa de la potencia del dispositivo eléctrico y del tiempo que dure operando, debido a esto, es necesario conocer el mayor desempeño de las bombas de calor (Arévalo et al., 2016). El desempeño de las bombas de calor se ve afectado por las propiedades físicas y térmicas de la fuente o sumidero de calor, así como del tipo de refrigerante utilizado. Además, el desempeño de los componentes tiene un papel importante en el coeficiente de operación de las bombas de calor. Existen muchos estudios e investigaciones que se centran en el desempeño y las condiciones de operación de los componentes de las bombas de calor, pero la mayoría se centra en el desempeño de un componente de la bomba de calor y sus efectos en su coeficiente de operación, o sea, investigan la interacción entre los componentes y todo el sistema de bomba de calor (Felten et al., 2018; Çakır y Çomaklı, 2016.). En este trabajo se realiza un análisis energético al equipo experimental que funciona como bomba de calor y enfriador de aire, TQ-Plint TE94, y se investigan los efectos que ocasiona el variar el flujo de agua suministrada.

\section{METODOLOGÍA}

Para realizar este trabajo, se utilizó el sistema TQ PLINT TE94, que consta de dos unidades separadas, el acondicionador de aire y la consola de control. Las dos unidades están conectadas por tuberías. El acondicionador de aire es completamente autónomo, y se compone de un sistema de refrigeración, un intercambiador de calor de aire y un intercambiador de agua, que se utilizan como fuente o sumidero según sea el funcionamiento (condensador o evaporador); un compresor; una válvula inversora de flujo, un ventilador, un colector de condensado, un manómetro inclinado y un tubo Pitot para medir el flujo de aire y un calentador de inmersión, como se muestran en la Fig. 1 y Fig.2.

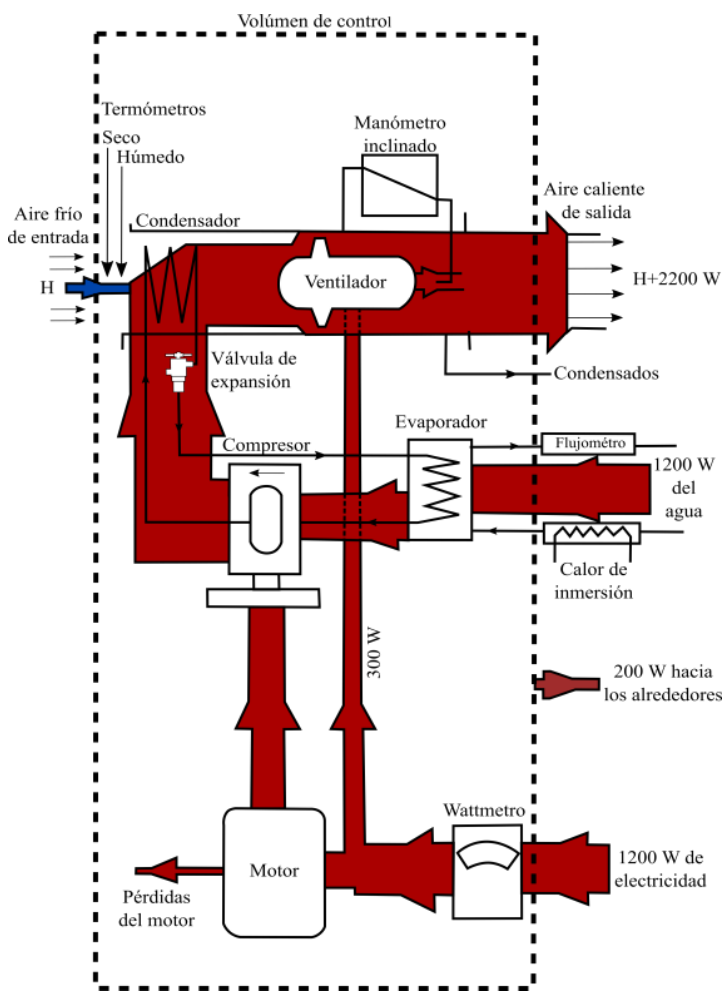

Fig. 1. Bomba de calor

Los instrumentos de medición contenidos en el panel de control son: un medidor de potencia para registrar la entrada de energía eléctrica al compresor y al ventilador; un medidor de temperatura multipunto que conecta a 10 termopares tipo k; un higrómetro para medir la humedad relativa del aire que entra y sale del sistema; un caudalímetro de agua; un vaso colector graduado para el condensado. El aire a acondicionar circula perpendicularmente en el 
intercambiador de calor de refrigerante R22, succionado por un ventilador, y finalmente el flujo de aire se descarga a un conducto circular.

En este trabajo, para determinar el desempeño del equipo se realizó un análisis energético en función del flojo volumétrico de agua, los flujos volumétricos de agua estudiados fueron $2,4,6$, y 8 L/min. La experimentación se realizó en la CDMX a las 9:00 horas, las condiciones ambientales fueron: Temperatura de 16-19 ${ }^{\circ} \mathrm{C}$, Humedad relativa del $60-85 \%$, y presión atmosférica de $77998 \mathrm{~Pa}$

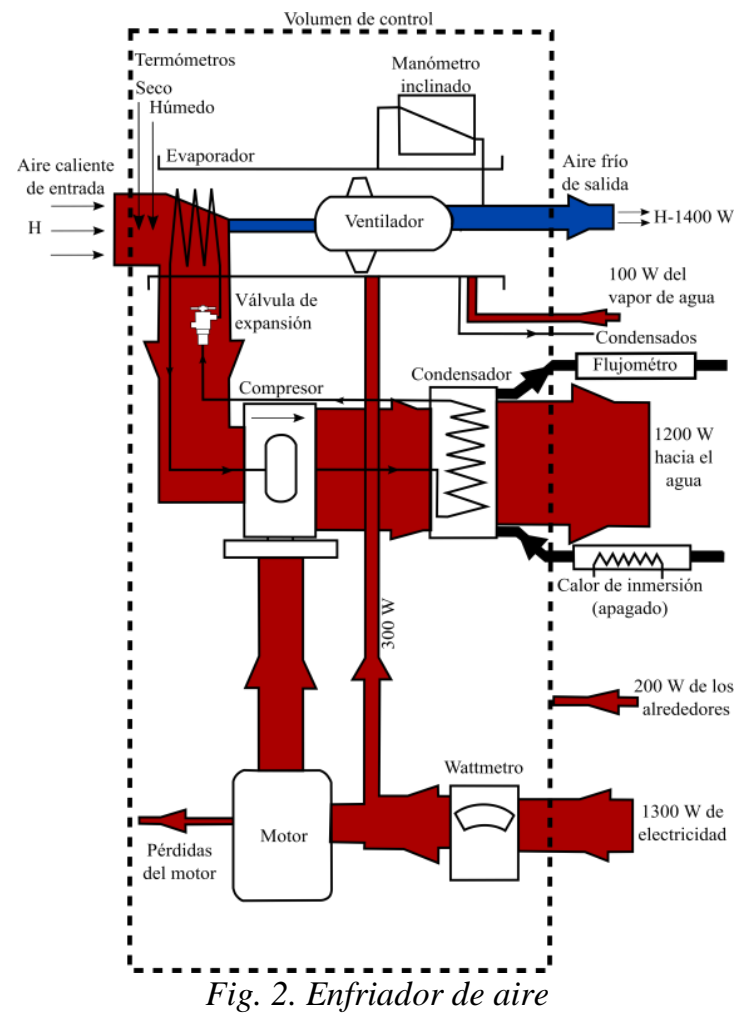

Cuando el equipo actúa como enfriador, la dirección del flujo ahora se invierte, el refrigerante pasa del compresor al intercambiador de calor de refrigerante-agua, es decir, pasa al condensador, en donde el refrigerante cede calor al agua, y enseguida pasa a través de la válvula de estrangulamiento, en donde disminuye su presión y temperatura; finalmente el refrigerante pasa a través del evaporador extrayendo el calor del aire y regresando al compresor. En este caso, el aire algunas veces se enfriará por debajo del punto de rocío y se depositará el condensado en el vaso colector graduado, en la Fig. 2 se muestra el diagrama esquemático del enfriador de aire. Los datos técnicos para el aire acondicionado Versatemp tipo vH250 son: Rango de Voltaje 220240 Volts; Corriente como Bomba de calor 5.5 Amperes; Corriente como enfriador 5.4 Amperes;
Potencia suministrada máxima $1.37 \mathrm{~kW}$; Potencia suministrada al calentador de inmersión $2 \mathrm{~kW}$; Flujo de agua de enfriamiento máximo $5 \mathrm{~L} / \mathrm{min}$ nominal; Flujo volumétrico de aire nominal 9 $\mathrm{m}^{3} / \mathrm{min}$; Capacidad nominal de calefacción/enfriamiento de 1.8 a $3.5 \mathrm{~kW}$. Por lo general, el acondicionador de aire se describe como un enfriador cuando extrae calor del aire circundante y lo rechaza al agua, y como una bomba de calor cuando transfiere calor en la dirección inversa, del agua al aire. Sin embargo, en ambos casos funciona como una máquina térmica invertida o una bomba de calor, ya que el efecto final es transferir calor de un depósito a otro, normalmente a una temperatura más alta, mediante el consumo de trabajo mecánico. El modelo teórico con el que se compara este dispositivo para evaluar su eficiencia, es la máquina reversible de Carnot. En la Máquina de Carnot se suministra el flujo de calor $\dot{Q}_{H}$ desde una fuente de alta temperatura $T_{H}$ a una máquina térmica, ésta produce un Potencia $\dot{\mathrm{W}}$, y rechaza un flujo de calor $\dot{Q}_{L}$ a un depósito que está a una temperatura baja $T_{L}$. En el Ciclo de Carnot todos los procesos son reversibles, entonces el ciclo se puede invertir y la máquina absorbería calor de la fuente fría y cedería calor a la fuente caliente, pero teniendo que suministrar potencia a la máquina. Si el objetivo de esta máquina es extraer calor de la fuente fría (para mantenerla fría) se denomina máquina frigorífica, y si es ceder calor a la fuente caliente, bomba de calor. Dicho motor absorbe calor desde una fuente a temperatura $T_{H}$, y lo rechaza a un depósito a una temperatura menor $T_{L}$, los procesos intermedios son isoentrópicos en el cual, $\mathrm{T}_{\mathrm{H}}>\mathrm{T}_{\mathrm{L}}$. El coeficiente de operación, $C O P$, de una bomba de calor real, se expresa como:

$$
C O P_{H}=\frac{\dot{Q}_{H}}{\dot{W}}
$$

y cuando opera como un enfriador:

$$
C O P_{R}=\frac{\dot{Q}_{L}}{\dot{W}}
$$

El coeficiente de operación máximo $(C O P)_{\max }$ de una bomba de calor ideal, es:

$$
\left(C O P_{H}\right)_{\max }=\frac{T_{H}}{T_{H}-T_{L}}
$$

y el de un enfriador

$$
\left(C O P_{R}\right)_{\max }=\frac{T_{L}}{T_{H}-T_{L}}
$$

También, para todos los casos se tiene que

$$
C O P_{H}=C O P_{R}+1
$$

Estas expresiones indican que, a medida que se reduce el tirante térmico entre la fuente fría y el 
sumidero caliente, el coeficiente de operación debe aumentar; una potencia determinada permite la transferencia de un flujo de calor desde la temperatura baja a una temperatura alta. En un sistema real, como el que se estudia en este trabajo, el coeficiente de operación es menor al ideal por las pérdidas eléctricas y mecánicas en el motor y compresor, la irreversibilidad del ciclo de enfriamiento en sí; la necesidad de diferencias de temperaturas entre el refrigerante y el aire, y entre el refrigerante y el agua, y como resultado el ciclo del refrigerante opera entre límites de temperaturas más amplios que los aplicables al agua y al aire que forman la fuente y el sumidero; y las pérdidas eléctricas y mecánicas en el ventilador. En la Fig. 3 , se muestran los diversos flujos de energía a través del sistema.

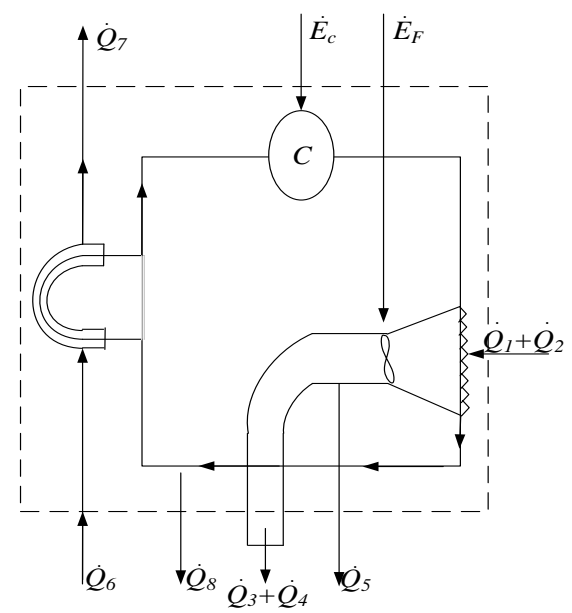

Fig. 3. Balance de energía en el sistema

Las expresiones para determinar los flujos de calor son:

-Entalpía por unidad de tiempo del aire seco a la entrada del acondicionador

$$
\dot{Q}_{1}=\dot{m}_{1} c_{P} T_{1}
$$

-El flujo másico de aire, $\dot{m}_{1}$ se expresa como:

$$
\dot{m}_{1}=0.00105 \sqrt{\frac{H_{1} P_{a}}{T_{2}}}
$$

-Entalpía por unidad de tiempo del vapor de agua a la entrada del acondicionador

$$
\dot{Q}_{2}=\omega \dot{m}_{1} h_{v}
$$

-Entalpía por unidad de tiempo del aire seco a la salida del acondicionador es

$$
\dot{Q}_{3}=\dot{m}_{1} c_{p} T_{2}
$$

-Entalpía por unidad de tiempo del vapor de agua a la salida del acondicionador

$$
\dot{Q}_{4}=h_{v}\left(\omega \dot{m}_{1}-\dot{m}_{2}\right)
$$

-Entalpía por unidad de tiempo del condensado

$$
\dot{Q}_{5}=\dot{m}_{2} h_{w}
$$

-Entalpía por unidad de tiempo del agua circulante en la entrada

$$
\dot{Q}_{6}=\dot{m}_{3} c_{P, w} T_{3}
$$

-Entalpía por unidad de tiempo del agua circulante a la salida

$$
\dot{Q}_{7}=\dot{m}_{3} c_{P, w} T_{4}
$$

-Pérdidas

$$
\dot{Q}_{8}
$$

-Entrada de potencia eléctrica al ventilador.

$$
\dot{E}_{F}
$$

La ecuación de flujo de energía para el sistema se puede escribir como sigue:

$$
\begin{aligned}
& \left(\dot{Q}_{6}-\dot{Q}_{7}\right)+\left(\dot{E}_{c}-\dot{E}_{F}\right)= \\
& \quad\left(\dot{Q}_{3}+\dot{Q}_{4}+\dot{Q}_{5}\right)-\left(\dot{Q}_{1}+\dot{Q}_{2}\right)+\dot{Q}_{8}
\end{aligned}
$$

El primer término representa el flujo calor suministrado al sistema desde el agua en circulación, y el segundo representa la entrada de energía eléctrica. La suma algebraica de estos términos se equipará con el aumento de flujo de calor del aire y el vapor de agua que pasa por el sistema, junto con las pérdidas; como se muestra en la Fig. 3. La ec. (14) se aplica por igual, con los cambios de señal apropiados, cuando la máquina está funcionando como enfriador. Cuando se opera como una bomba de calor, el coeficiente de operación se puede definir de dos maneras diferentes. El coeficiente de operación total externo es:

$$
\left(\mathrm{COP}_{H}\right)_{E}=\frac{\left(\dot{Q}_{3}+\dot{Q}_{4}+\dot{Q}_{5}\right)-\left(\dot{Q}_{1}+\dot{Q}_{2}\right)}{\left(\dot{E}_{c}+\dot{E}_{F}\right)}
$$

El coeficiente de operación correspondiente para una bomba ideal que opera entre las mismas temperaturas medias es:

$$
\left(C O P_{H}\right)_{\max }=\frac{\frac{1}{2}\left(T_{1}+T_{2}\right)}{\frac{1}{2}\left(T_{1}+T_{2}\right)-\frac{1}{2}\left(T_{3}+T_{4}\right)}
$$

El coeficiente de operación de la bomba de calor básica no es idéntico al coeficiente de operación general, ya que la potencia suministrada al ventilador no interviene en el ciclo del refrigerante y la del compresor sí. Esto conduce a un coeficiente de operación interno:

$$
\left(C O P_{H}\right)_{I}=\frac{\left(\dot{Q}_{3}+\dot{Q}_{4}+\dot{Q}_{5}\right)-\dot{E}_{F}-\left(\dot{Q}_{1}+\dot{Q}_{2}\right)}{\left(\dot{E}_{c}\right)}
$$

Esto se puede comparar con el coeficiente de operación ideal basado en la diferencia de 
temperatura a través del circuito de la bomba de calor:

$$
\left(C O P_{H}\right)_{I}=\frac{T_{10}}{T_{10}-T_{8}}
$$

De manera similar, se pueden reconocer dos criterios diferentes cuando el sistema está funcionando como enfriador:

$$
\left(C O P_{R}\right)_{E}=\frac{\left(\dot{Q}_{1}+\dot{Q}_{2}\right)-\left(\dot{Q}_{3}+\dot{Q}_{4}+\dot{Q}_{5}\right)}{\left(\dot{E}_{c}+\dot{E}_{F}\right)}
$$

en comparación con el ideal

$$
\left(C O P_{R}\right)_{\max }=\frac{\frac{1}{2}\left(T_{1}+T_{2}\right)}{\frac{1}{2}\left(T_{3}+T_{4}\right)-\frac{1}{2}\left(T_{1}+T_{2}\right)}
$$

$\mathrm{y}$

$$
\left(C O P_{R}\right)_{I}=\frac{\left(\dot{Q}_{1}+\dot{Q}_{2}\right)+\dot{E}_{F}-\left(\dot{Q}_{3}+\dot{Q}_{4}+\dot{Q}_{5}\right)}{\dot{E}_{c}}
$$

en comparación con el ideal

$$
\left(C O P_{R}\right)_{\max }=\frac{T_{10}}{T_{8}-T_{10}}
$$

En el caso del enfriador, $T_{8}$ es la temperatura a la salida del intercambiador refrigerante-agua, y $T_{10}$ es la temperatura de entrada del intercambiador refrigerante-aire.

Las propiedades termodinámicas del aire húmedo han sido objeto de un minucioso estudio desde mediados del siglo pasado, debido a su fundamental importancia para el diseño y construcción de sistemas de aclimatación de locales, tanto si es necesario enfriarlos en climas tropicales o si es necesario calentarlos en climas fríos (Balbis et al., 2018).

En este trabajo, la humedad específica del aire que ingresa al acondicionador está relacionada con la humedad relativa $\phi$ medida por el higrómetro digital:

$$
\omega=0.622\left(\frac{\phi P_{\text {sat }}}{P_{\text {atm }}-\phi P_{\text {sat }}}\right)
$$

donde, $P_{\text {sat }}$ y $P_{\text {atm }}$ son la presión de saturación y la presión atmosférica a la temperatura de entrada del aire en el acondicionador.

\section{ANÁLISIS DE RESULTADOS}

Para evaluar el sistema TQ PLINT TE94, enfriador de aire-bomba de calor, se realizan variaciones del flujo volumétrico de agua en el intercambiador de calor, del agua de enfriamiento con el refrigerante $\mathrm{R} 22$. Los datos obtenidos para el enfriador de aire y bomba de calor se resumen en las Tablas 1 y 2 , respectivamente.

Tabla 1. Pruebas en el enfriador de aire

\begin{tabular}{ccccc} 
& \multicolumn{4}{c}{$\begin{array}{c}\text { Flujos volumétricos de agua en el } \\
\text { intercambiador de calor }\end{array}$} \\
\cline { 2 - 5 } & $2 \mathrm{~L} / \mathrm{min}$ & $4 \mathrm{~L} / \mathrm{min}$ & $6 \mathrm{~L} / \mathrm{min}$ & $8 \mathrm{~L} / \mathrm{min}$ \\
\hline $\boldsymbol{T}_{\boldsymbol{1}}\left({ }^{\circ} \mathbf{C}\right)$ & 19 & 17 & 17 & 16 \\
$\boldsymbol{T}_{2}\left({ }^{\circ} \mathbf{C}\right)$ & 14 & 13 & 13 & 13 \\
$\boldsymbol{T}_{\mathbf{3}}\left({ }^{\circ} \mathbf{C}\right)$ & 26 & 30 & 31 & 32 \\
$\boldsymbol{T}_{\boldsymbol{4}}\left({ }^{\circ} \mathbf{C}\right)$ & 48 & 39 & 37 & 37 \\
$\boldsymbol{T}_{5}\left({ }^{\circ} \mathbf{C}\right)$ & 2 & 1 & 1 & 0 \\
$\boldsymbol{T}_{\boldsymbol{6}}\left({ }^{\circ} \mathbf{C}\right)$ & 70 & 66 & 65 & 66 \\
$\boldsymbol{T}_{7}\left({ }^{\circ} \mathbf{C}\right)$ & 70 & 66 & 65 & 66 \\
$\boldsymbol{T}_{\boldsymbol{s}}\left({ }^{\circ} \mathbf{C}\right)$ & 36 & 32 & 32 & 32 \\
$\boldsymbol{T}_{\boldsymbol{9}}\left({ }^{\circ} \mathbf{C}\right)$ & 2 & 1 & 0 & 0 \\
$\boldsymbol{T}_{\boldsymbol{1 0}}\left({ }^{\circ} \mathbf{C}\right)$ & 3 & 2 & 1 & 1 \\
$\dot{\boldsymbol{E}}_{\boldsymbol{c}}(\mathbf{W})$ & 683 & 680 & 685 & 701 \\
$\dot{\boldsymbol{E}}_{\boldsymbol{f}}(\mathbf{W})$ & 447 & 430 & 385 & 359 \\
$\boldsymbol{P}_{\boldsymbol{a}}(\mathbf{P a})$ & 77,998 & 77,998 & 77,998 & 77,998 \\
$\phi_{\text {ent }}(\mathbf{\%})$ & 67 & 89 & 86 & 83 \\
$\phi_{\text {sal }}(\mathbf{\%})$ & 54 & 80 & 67 & 66 \\
$\dot{\boldsymbol{m}}_{\boldsymbol{2}}(\mathbf{k g} / \mathbf{s})$ & 0.00029 & 0.00083 & 0.00045 & 0.00056 \\
\hline
\end{tabular}

Tabla 2. Pruebas en la bomba de calor Flujos volumétricos de agua en el intercambiador de calor

\begin{tabular}{ccccc} 
& \multicolumn{4}{c}{ intercambiador de calor } \\
\cline { 2 - 5 } & $2 \mathrm{~L} / \mathrm{min}$ & $4 \mathrm{~L} / \mathrm{min}$ & $6 \mathrm{~L} / \mathrm{min}$ & $8 \mathrm{~L} / \mathrm{min}$ \\
\hline $\boldsymbol{T}_{\boldsymbol{1}}\left({ }^{\circ} \mathbf{C}\right)$ & 25 & 27 & 28 & 28 \\
$\boldsymbol{T}_{2}\left({ }^{\circ} \mathbf{C}\right)$ & 45 & 48 & 50 & 51 \\
$\boldsymbol{T}_{3}\left({ }^{\circ} \mathbf{C}\right)$ & 18 & 16 & 16 & 15 \\
$\boldsymbol{T}_{\boldsymbol{4}}\left({ }^{\circ} \mathbf{C}\right)$ & 7 & 11 & 12 & 12 \\
$\boldsymbol{T}_{5}\left({ }^{\circ} \mathbf{C}\right)$ & 73 & 86 & 89 & 91 \\
$\boldsymbol{T}_{\boldsymbol{6}}\left({ }^{\circ} \mathbf{C}\right)$ & 2 & 6 & 7 & 8 \\
$\boldsymbol{T}_{7}\left({ }^{\circ} \mathbf{C}\right)$ & 2 & 6 & 7 & 8 \\
$\boldsymbol{T}_{8}\left({ }^{\circ} \mathbf{C}\right)$ & 11 & 14 & 15 & 16 \\
$\boldsymbol{T}_{\boldsymbol{9}}\left({ }^{\circ} \mathbf{C}\right)$ & 73 & 86 & 89 & 91 \\
$\boldsymbol{T}_{10}\left({ }^{\circ} \mathbf{C}\right)$ & 42 & 50 & 51 & 52 \\
$\dot{\boldsymbol{E}}_{\boldsymbol{c}}(\mathbf{W})$ & 877 & 950 & 941 & 917 \\
$\dot{\boldsymbol{E}}_{\boldsymbol{f}}(\mathbf{W})$ & 363 & 370 & 409 & 453 \\
$\boldsymbol{P}_{\boldsymbol{a}}(\mathbf{P a})$ & 77,998 & 77,998 & 77,998 & 77,998 \\
$\boldsymbol{\phi}_{\text {ent }}(\mathbf{( \% )}$ & 35 & 25 & 34 & 41 \\
$\phi_{\text {sal }}(\mathbf{\%})$ & 11 & 10 & 10 & 10 \\
\hline
\end{tabular}

La Fig. 4 muestra el diagrama presión-entalpía del enfriador de aire en el circuito del refrigerante R22, para un flujo volumétrico de agua de $2 \mathrm{~L} / \mathrm{min}$ y 6 $\mathrm{L} / \mathrm{min}$. El efecto de enfriamiento, $h_{1}-h_{4}$, es mayor para un flujo de $6 \mathrm{~L} / \mathrm{min}$; al aumentar el flujo de agua de $2 \mathrm{~L} / \mathrm{min}$ a $6 \mathrm{~L} / \mathrm{min}$ ocasiona un incremento del $2.97 \%$ en el efecto de enfriamiento. Mientras el trabajo suministrado al compresor, $h_{2}-h_{1}$, disminuye $5.68 \%$ al incrementar el flujo volumétrico de agua de 2 a $6 \mathrm{~L} / \mathrm{min}$. Entonces, con relación al efecto de enfriamiento y el trabajo de compresión, el coeficiente de operación del circuito refrigerante es mayor cuando el flujo de agua es de $6 \mathrm{~L} / \mathrm{min}$. 


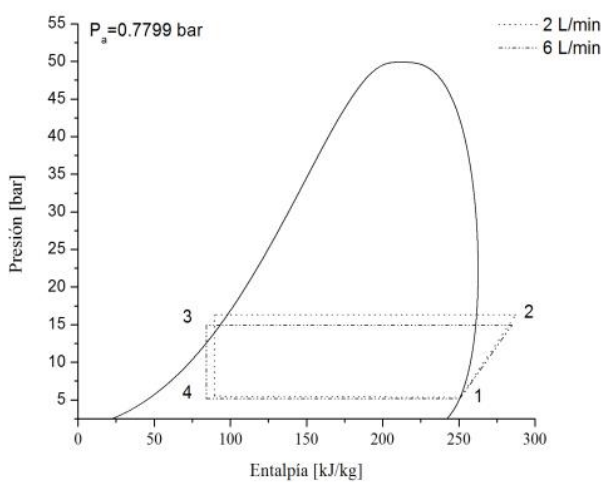

Fig. 4. Diagrama Presión-Entalpía del enfriador de aire, a diferentes flujos volumétricos de agua

La Fig. 5 muestra el diagrama Presión-Entalpía de la bomba de calor en el ciclo del refrigerante R22, para un flujo volumétrico de agua de $2 \mathrm{~L} / \mathrm{min}$ y 6 $\mathrm{L} / \mathrm{min}$. El efecto de calentamiento, $h_{2}-h_{3}$, es mayor para el gasto de $2 \mathrm{~L} / \mathrm{min}$ que para el gasto de 6 $\mathrm{L} / \mathrm{min}$ en $2.64 \%$. Además, el trabajo suministrado en el compresor, $h_{2}-h_{1}$, es menor con el flujo volumétrico de agua de $2 \mathrm{~L} / \mathrm{min}$ que con el de 6 $\mathrm{L} / \mathrm{min}$ en un $14.11 \%$. Por consiguientes, el coeficiente de operación del equipo es mayor para el flujo volumétrico de agua de $2 \mathrm{~L} / \mathrm{min}$.

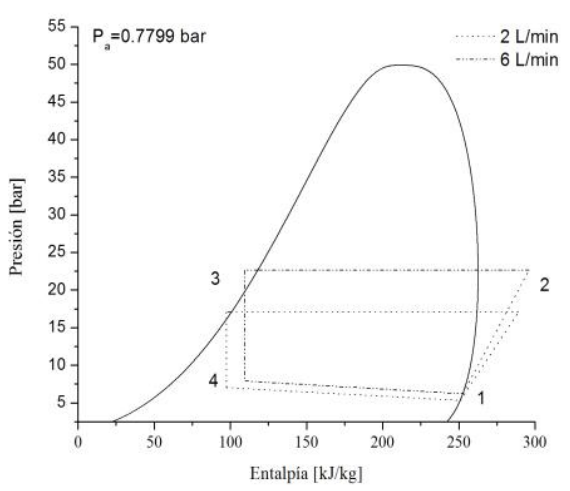

Fig. 5. Diagrama Presión-Entalpía de la bomba de calor, para diferentes flujos volumétricos de agua

La Fig. 6 muestra el comportamiento de la potencia suministrada al enfriador de aire en función del flujo volumétrico del agua. $\mathrm{Si}$ el flujo de agua aumenta de $2 \mathrm{~L} / \mathrm{min}$ a $8 \mathrm{~L} / \mathrm{min}$, la potencia total suministrada al enfriador de aire presenta una disminución del $6.19 \%$, debido a que la potencia del ventilador disminuye $19.68 \%$, a pesar de que la potencia del compresor aumenta $2.63 \%$. Además, el comportamiento de la potencia del compresor y del flujo másico del refrigerante son semejantes; en este caso, la mínima potencia suministrada al compresor es de $680 \mathrm{~W}$ y es para un flujo volumétrico de agua de $4 \mathrm{~L} / \mathrm{min}$, debido a que también tiene el menor flujo másico de refrigerante, que es de $12.49 \mathrm{~kg} / \mathrm{s}$.

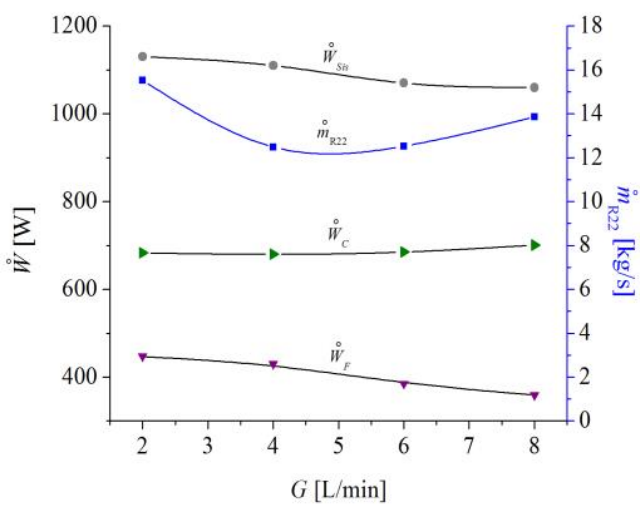

Fig. 6. Potencias suministradas al enfriador de aire en función del flujo volumétrico de agua

La Fig. 7 muestra el comportamiento de la potencia suministrada a la bomba de calor en función del flujo volumétrico del agua. Cuando el flujo volumétrico se incrementa de $2 \mathrm{~L} / \mathrm{min}$ a $8 \mathrm{~L} / \mathrm{min}$, la potencia total suministrada a la bomba de calor se incrementa el $10.48 \%$, debido a que la potencia consumida por el ventilador se incrementa el 24.79 $\%$; sin embargo, para el rango de $4 \mathrm{~L} / \mathrm{min}$ a 8 $\mathrm{L} / \mathrm{min}$, la potencia del compresor disminuye el 3.47 $\%$. Además, el comportamiento de la potencia del compresor es contrario a la del flujo de refrigerante, es decir, cuando la potencia del compresor aumenta, el flujo de refrigerante disminuye y viceversa. La potencia suministrada mínima al compresor es de $877 \mathrm{~W}$ para un flujo volumétrico de agua de $2 \mathrm{~L} / \mathrm{min}$, debido a que el trabajo de compresión es de $38.04 \mathrm{~kJ} / \mathrm{kg}$ (como se muestra en la Fig. 6), a pesar que el flujo de refrigerante a esa condición no sea el menor.

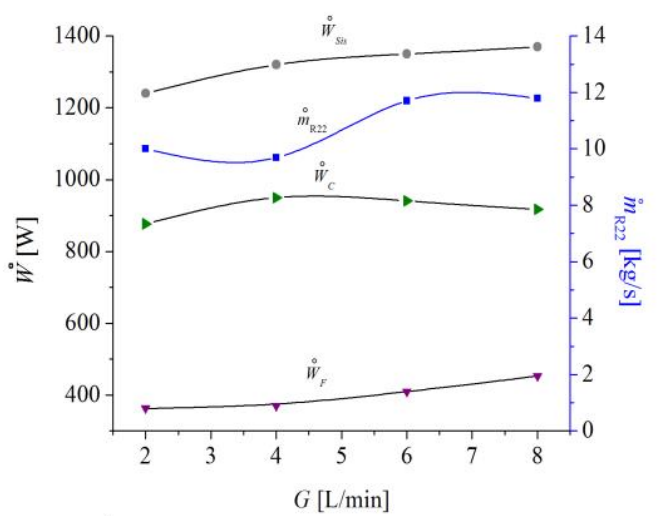

Fig. 7. Potencias suministradas a la bomba de calor en función del flujo volumétrico de agua 
La Fig. 8 muestra los diferentes coeficientes de operación del enfriador de aire para el rango estudiado del flujo volumétrico de agua, de 2 a 8 L/min. Los coeficientes de operación internos son mayores a los coeficientes de operación externos, ya que los coeficientes de operación externos consideran la potencia del ventilador, sin embargo, para los COP's reales (interno y externo), el comportamiento es similar, es decir, sus curvas de comportamiento son casi paralelas. Además, se tiene que los coeficientes de operación ideal interno, real interno y real externo presentan los mayores valores en un flujo volumétrico de agua de $4 \mathrm{~L} / \mathrm{min}$, con $9.17,4.35$ y 2.28 respectivamente; esta situación se debe a que la potencia suministrada al compresor y del flujo másico de refrigerante son menores. $\mathrm{Y}$ el mayor coeficiente de operación ideal externo es de 15.16 y se tiene para un flujo de $6 \mathrm{~L} / \mathrm{min}$.

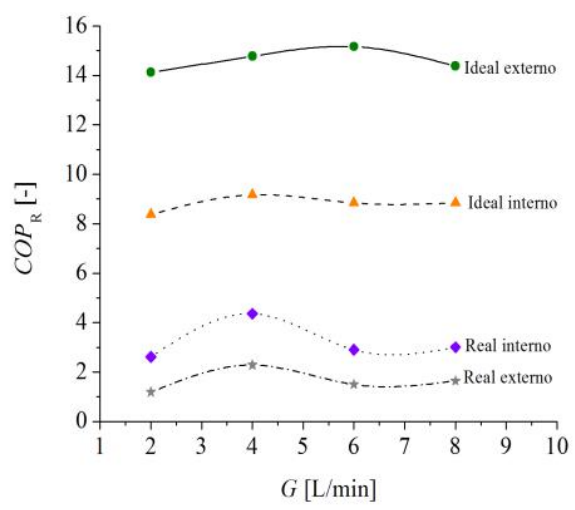

Fig. 8. Coeficientes de operación del enfriador de aire en función del flujo volumétrico del agua

La Fig. 9 muestra los diferentes coeficientes de operación de la bomba de calor con respecto al flujo volumétrico de agua. Al igual que el enfriador de aire, los coeficientes de operación externos son menores a los coeficientes de operación internos, así como los COP's reales (interno y externo) tienen comportamientos similares y se encuentran muy cercanos, por ejemplo, para un flujo de agua de $6 \mathrm{~L} / \mathrm{min}$, la diferencia entre el COP interno y COP externo es de 0.3. Además, los mayores COP's reales (interno y externo) se tienen para un flujo volumétrico de $8 \mathrm{~L} / \mathrm{min}$, con 2.10 y 1.73 , respectivamente. Por otra parte, para un flujo de 4 $\mathrm{L} / \mathrm{min}$, el COP ideal externo es $87.2 \%$ mayor que el COP real externo; el COP ideal interno es 78.7 $\%$ mayor que el COP real interno; y el COP real interno es $13.36 \%$ mayor con respecto al COP real externo.

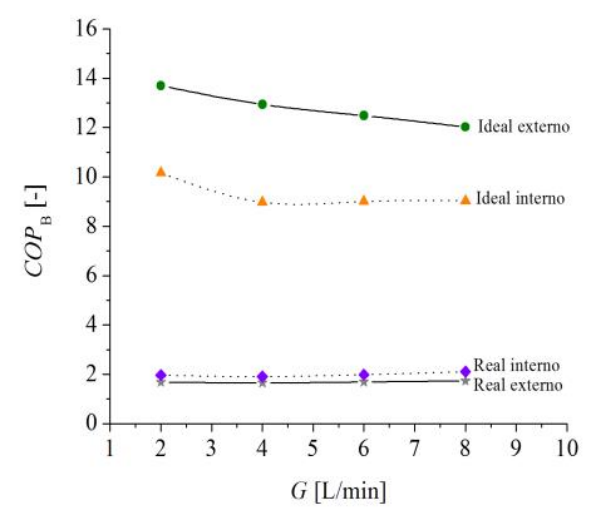

Fig. 9. Coeficientes de operación de la bomba de calor en función del flujo volumétrico del agua

\section{CONCLUSIONES}

Los coeficientes de operación externos son menores a los coeficientes de operación internos, debido a que los primeros consideran la potencia del ventilador junto con la potencia del compresor como entradas requeridas, para ambos casos. Los COP's reales (interno y externo) tienen comportamientos similares. Para el enfriador de aire, la potencia suministrada al compresor tiene el mismo comportamiento que el flujo másico del refrigerante. Por otra parte, la menor potencia suministrada al compresor es de $680 \mathrm{~W}$, y es para un flujo volumétrico de agua de $4 \mathrm{~L} / \mathrm{min}$, y además se tiene el menor flujo másico de refrigerante con $12.49 \mathrm{~kg} / \mathrm{s}$, y por ende, se tienen los mayores coeficientes de operación reales en el enfriador de aire. Para la bomba de calor, la potencia suministrada al compresor tiene un comportamiento contrario al flujo másico de refrigerante, es decir, si la potencia del compresor se incrementa, el flujo de refrigerante disminuye y viceversa. Además, la menor potencia suministrada al compresor es de $877 \mathrm{~W}$ y es para un flujo volumétrico de agua de $2 \mathrm{~L} / \mathrm{min}$, debido a que el trabajo de compresión es de $38.04 \mathrm{~kJ} / \mathrm{kg}$, a pesar de que el flujo de refrigerante a esa condición no sea el menor, en consecuencia, se tienen los mayores coeficientes de operación reales en la bomba de calor.

\section{REFERENCIAS}

Chua, K. J.; Chou, S. K.; Yang, W. M. (2010). "Advances in heat pump systems: A review". Applied Energy, Vol. 87, pp. 3611-3624.

C. J. Torres, J. F. Archila, M. L. Tronco, M. Becker, A. J. V. Porto, A. J. Tiberti. (2013). Estudio cinemático de una plataforma robótica para agricultura. REVISTA 
COLOMBIANA DE TECNOLOGÍAS DE AVANZADA, ISSN: 1692-7257. 2(22).

Torres, C., Archila, J., Tronco, M., Becker, M., Viera Porto, A., \& Tiberti, A. (2013). Estudio cinemático de una plataforma robótica para agricultura. REVISTA COLOMBIANA DE TECNOLOGÍAS DE AVANZADA, 2(22).

Saloux, E.; Sorin, M.; Teyssedou, A. (2018). "Assessing the exergy performance of heat pump systems without using refrigerant thermodynamic properties". International Journal of Refrigeration, Vol. 98, pp. 1-9.

F. A.L. González, M. M. H. Cely. (2013). Estudio del potencial eólico y solar de Cúcuta, Norte de Santander. REVISTA COLOMBIANA DE TECNOLOGÍAS DE AVANZADA, ISSN: 1692-7257. 2(22).

Leal González, F., \& Hernandez Cely, M. (2013). Estudio del potencial eólico y solar de Cúcuta, Norte de Santander. REVISTA COLOMBIANA DE TECNOLOGÍAS DE AVANZADA, 2(22).

Felten, B.; Weber, C. (2018). "The value(s) of flexible heat pumps-Assessment of technical and economic Conditions". Applied Energy, Vol. 228, pp. 1292-1319.

Çakir, U.; Çomakli, K. (2016). "Exergetic interrelation between and heat pump and components". Applied Thermal Engineering, Vol. 105, pp. 659-668.

Balbis M., García R. (2018). "Caracterización energética del funcionamiento de un equipo de aire acondicionado en un local dado". Revista Colombiana de Tecnologías de Avanzada, Vol. 2, pp. 86-91.

Arévalo T., Cabellos M., García R. (2016). "Consumo de energía eléctrica en diferentes viviendas en la Ciudad de Ocaña, Norte de Santander". Revista Colombiana de Tecnologías de Avanzada, Vol. 1, pp. 42-46.

\section{ANEXOS}

\section{Nomenclatura}

$c_{P} \quad$ calor específico del aire a presión constante; $\left[\mathrm{J} / \mathrm{kg}^{\circ} \mathrm{C}\right]$,

$c_{P, w} \quad$ calor específico del agua; $\left[\mathrm{J} / \mathrm{kg}^{\circ} \mathrm{C}\right]$,

$\mathrm{G}$ gasto; [L/min],

$h_{v} \quad$ entalpía específica de vapor de agua; $[\mathrm{J} / \mathrm{kg}]$,

$H_{w}$ entalpía específica de condensado; $[\mathrm{J} / \mathrm{kg}]$,

$\dot{m}_{1} \quad$ flujo másico de aire seco; $[\mathrm{kg} / \mathrm{s}]$,

$\dot{m}_{2}$ flujo másico de condensado; [ $\left.\mathrm{kg} / \mathrm{s}\right]$,

$\dot{m}_{3}$ flujo másico de circulación de agua; [kg/s],
$P_{\text {atm }}$ presión atmosférica; $[\mathrm{Pa}]$,

$T_{1}$ temperatura de aire en la entrada; [K],

$T_{2}$ temperatura de aire en la descarga; [K],

$T_{3}$ temperatura de circulación de agua en la entrada; $[\mathrm{K}]$,

$T_{4}$ temperatura de circulación de agua en la descarga; $[\mathrm{K}]$,

Bomba de calor / Enfriador

$T_{5 . \ldots . . .}$ compresor; [K], Descarga / Entrada

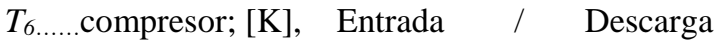

$T_{7}$ refrigerante en el intercambiador de calor con agua; $[\mathrm{K}], \quad$ Entrada / Descarga

$T_{8}$ refrigerante en el intercambiador de calor con agua; [K] Entrada / Descarga

$T_{9}$ intercambiador de calor con refrigerante con aire; [K] Entrada / Descarga

$T_{10}$ intercambiador de calor con refrigerante con aire; $[\mathrm{K}] \quad$ Descarga / Entrada 